\title{
Health and Aging of the Elderly in India
}

\author{
Radhika Kapur* \\ Pedagogy and Organizational Culture in Nursery Schools, Delhi University, New Delhi, India \\ *Corresponding Author: Radhika Kapur, Pedagogy and Organizational Culture in Nursery Schools, Delhi University, New Delhi, India.
}

Received: August 19, 2019; Published: September 26, 2019

Old age manifests greater diversity than any other age group. Aging can be understood through the interpretation of the individual psychological state of mind, physical health, viable and prospective economic conditions, retaining status, level of activity and importance of place within the social environment. The term "aging" is meant to denote determinate patterns of late changes, it is not merely older but old. Old age is characterized by gradual changes in appearances such as graying of hair, loss of hair, teeth, elongation of nose, ear, sagging skins, wrinkles, impairment of sensory functions such as vision, audition, smelling, tactility, feeling etc. Aging leads to diminishing capacity of physical work and sexual arousal is becoming more and more prominent.

Mental health is not only related to the aging of the brain but also socio-economic factors such as helplessness, lack of selfconfidence and solitude. In India, aging is considered to be negative aging, where elderly adopt a pessimistic attitude towards life. The absence of social support network, indifferent attitude of the caretakers, increased dependency, physical illness, unhappiness, loss of activities from life, and many more are responsible for the poor self-rated mental health and life satisfaction. Loneliness is a chronic and a distressful mental state; older persons often feel neglected, marginalized, alienated and helpless. Social support has a significant role in making the elderly feel wanted and supported; interaction and communication with the other people regarding their concerns and matters make them feel relieved. Social support constitutes not just family members but also friends, neighbors, and co-workers.

During the old age, if an individual is surrounded by social support network of neighbors, friends and other people they feel satisfied, if husband and wife support each other they feel satisfied. In other words, when they have good means of communication and interaction with the society they incur the feeling of life satisfaction. In most affluent countries and areas, financial security for the elderly can in principle be achieved through impersonal mechanisms, such as pension schemes and private or government transfers, but reliance on families to care for elderly members who are dependent upon others is likely to prove increasingly problematic.

In the Asian countries, there has been an increase in the elderly population and women are suffering from anxiety and depression more as compared to men. How older men and women differ from each other as concerning their social, physical, cultural and solitary activities, social support and life satisfaction is on the basis of gender differences. The mindset of the older men does differ as compared to the older women.

Perception of their physical health and opinion regarding the future is a very important aspect for the elderly, and this depends upon mental health. Those who look upon aging with a pessimistic opinion are likely to suffer from shortcomings related to social, economic, physical and behavior and those who look upon aging with a positive mindset seem to be aging gracefully. There have been certain factors which have a direct bearing upon the mental health system of the elderly: a) disruption of family support due to industrialization and urbanization, b) empty-nest phenomenon, c) decrease in economic and financial status and d) loss of a distant relative or a loved one. Among the elderly population, males have better physical fitness than the females. Exercise and physical activity also help the elderly to achieve a sense of purpose. The come to realize that they have more control over their body then they had imagined. 
In order to ensure a more productive and less restrictive old age for women there are the following features/steps to be undertaken:

1. Education for women.

2. Awareness of the aging process and its physiological implications.

3. Information about the various preventive medicines and health care systems.

4. In India, the joint family system is being replaced by the nuclear family system. Joint family is the family where two or more nuclear families stay under the common roof with unmarried aunts and uncles too living there. It is advantageous to have a joint family system where care and warmth is there. On the other hand, nuclear family comprises of husband-wife and their children, elderly should be helped to find care and warmth in a nuclear family as well, therefore, it is vital to help the elderly people see the positive side of the nuclear family system

5. "Empty nest" syndrome leads to depression and poor health in women, an alternative to this would be productive occupation of leisure time.

Gerontology is the scientific study of the human aging process. One quarter of the life is spent in growing up and three-quarters in growing old. The phenomenon of population aging is due to increased life-expectancy and falling death rate. The changes can be attributed to better environmental conditions, socio-economic progress, healthier lifestyle, increasing availability of high quality medical care and large scale introduction of a wide range of life saving drugs. The population has gained at least 30 years of life expectancy in India.

\section{Health of the elderly in winter season}

The health of the elderly persons is affected by the weather conditions too, extremely hot or extremely cold temperatures do affect the health of the elderly persons. Winter season is the season of merriment; it's also the season for hypothermia, slips, falls and flu. The elderly are especially susceptible to winter trauma and it is extremely important to be aware of the risks they face. There are certain steps that need to be taken care of and these are as follows:

1. Test the Weather: Before charging out of the front door it is crucial to test the weather to see how cold it is or it is windy. It is vital to get the feel of the weather before heading out.
2. Re-adjust Schedule: If it's cold, windy or cloudy, then for the maintenance of good health it is crucial to stay indoors and reschedule appointments and other work related concerns.

3. Lighting: On the streets it is important to ensure that good lighting is there or if the street lighting facility is not present than it is mandatory to use flashlight.

4. Appropriate Attire: It is important to wear warm clothing during the winter season. Layer clothing is best as it insulates best. Head cover, gloves, socks are a must when going out. Lack of appropriate attire may enable them to fall sick.

5. Proper Nutrition: Winter season means lots of binging upon cakes and goodies. For the elderly people it is required to consume regular nutritious diet. This will provide them energy and strength.

6. Vision and Assistive Devices: Elderly persons are required to get their vision checked regularly and keep canes and walkers in order to remain ambulatory, especially outside the homes. Sometimes elderly may go out alone for a walk or to the mall, then it is required for them to take their spectacles and canes; failing to take these devices may cause accidents.

Older adults are at a greater risk because the body's ability to maintain a constant internal temperature decreases with age. Slips and falls occur more commonly in the winter months because the body is stiff. Older adults are at the greatest risk of getting complications from flu, such as pneumonia and dehydration. They have diminished cough and gag reflexes, and their immune systems are also not very strong.

\section{Marital status of the elderly}

Among the elderly population in India, married elderly persons seem to fare better economically, socially, financially as compared to those who are single. There have been an increasing proportion of elderly women, amongst the population of India; most of the elderly women are widows. There have been two reasons for this, firstly, longer lifespan of women as compared to men and secondly, women marry the men generally older than themselves. Married men and women normally are contented and satisfied; they feel relieved from the feeling of loneliness. On the other hand, widowhood is extremely difficult for an elderly person; he/she is bounded by depression and loneliness.

At the stage of widowhood, mostly children do care for their parents and keep their parents with them; on the other hand, some 
children live abroad, but keep visiting their parents in India in order to take care of them. In India, there are some of the children who are warped minded and do not like to look after their parents, they ill treat them for the sake of property and finances, they send them to old age homes and misbehave with their parents. This tends to develop a pessimistic viewpoint in the minds of the elderly people towards life. These inappropriate measures have been largely discouraged in India, with the help of organizations. Help Age India is the country's largest voluntary organization with 23 regional offices. It has supported 1600 projects all over the country. Besides national level voluntary organizations, a number of regional and local level NGOs have set up multi-services facilities and innovative programs. Some of the examples are Action for Social Help Assistance (ASHA), Family Welfare Agency, Dignity Foundation, Meals on Wheels and Development Welfare and Research Foundation (DWARF) all operating in different parts of the country. Their activities include providing second careers, income generating activities, companionship, cooked meals, nutritional counseling, help-line services and promoting active aging.

Living arrangements among the elderly

The conventional living patterns among the elderly have changed drastically following the reduction in fertility and the increase in life expectancy at older ages. In India, the traditional practice has been for people to live with their children in old age; this is not necessarily with the intention of receiving support; often the rest of the family also benefits from arrangement. For example, when the younger women of the household go to work, the grandparents take care of their children.

The term "living arrangement" refers to ones household structure, it refers to the type of family in which the elderly live, the headship that they get pleasure from, the place they stay in, the people they stay with, the kind of relationship that they maintain with their kith and kin and the extent to which they adjust to the changing environment. The elderly people are normally less independent and often require care and support of other individuals in several respects. Taking care of the elderly refers mainly to emotional support; on the other hand, support that is rendered to the elderly refers to financial and material support. Emotional support is expected from family members or other close relatives, whereas, financial and material support is supposed to be the joint effort of the immediate family and society.
Role of criminal justice functionaries and NGOs in welfare of elderly people

In India, almost $30 \%$ of the elderly are subject to some form of abuse or neglect by their families. Financial exploitation occurs when the offender steals, withholds, or otherwise misuses their elderly victim's money, property or valuables for personal advantage or profit, to the disadvantage of the elderly. Their methods can include: 1) simply taking money, valuables etc, 2) borrowing and not paying back, 3) denying services or medical care to conserve funds, 4) giving away or selling possessions without permission, 5) misusing ATM/credit cards, 6) signing or cashing pension without permission and 7) forcing the elderly to part with resources or sign over property.

One of the reasons of crime against elderly is their lack of information. Physical weakness, social isolation, lack of care from children, and dependency on hired domestic help has made them more vulnerable to violent crimes. NGOs can play a very significant role in providing the necessary information and support. They make provision of invaluable service by providing them facilities at their doorstep. Assessment of the victims is an important contribution made by the NGOs, another contribution is reducing the isolation of the elderly through welfare checks, and in-person outreach efforts can help to ensure that the elders are aware of available resources that they can turn to in time of need. Adopt-a-senior, or adopt -an-area are also effective programs. Criminal justice functionaries identify areas of greater vulnerability like colonies near slum areas, increase patrolling of colonies where residents are mostly elderly persons; visiting homes of elderly to ensure safety precautions, elderly can report their abuse and exploitation also due to home visiting. They help the elderly to coordinate in getting justice in an easier and faster manner.

\section{Discussion}

In India, the elderly population is less independent and more dependent upon family members and other relatives for their existence. Majority of the children do take care of their parents but $30 \%$ of the elderly people are subject to some form of abuse such as physical, sexual, psychological, financial, social, environmental and self-neglect. Criminal Justice Functionaries and NGOs do form networking and co-ordination in the welfare of the elderly people. With respect to the weather conditions, it is important for 
the elderly people to take effective precautions, especially during the cold. Lack of warm clothing can upset their health conditions. Physical exercise, nutritious diet, socialization with family and friends and obtaining information through newspapers, radio, television and so forth can highly assist them in living a healthy and a contented life.

Volume 3 Issue 10 October 2019

(C) All rights are reserved by Radhika Kapur. 